\title{
Über die Darstellung von reinem Molybdän.
}

\author{
Von \\ Ad. VANDENBERGHe. ${ }^{1}$ \\ Mit 1 Figur im Text.
}

Unter den veröffentlichten Methoden zur Gewinnung reinen Molybdäns sind einige ganz mangelhaft, aber es sind zwei solche bekannt, die auf den ersten Blick den Anschein geben, als mürsten sie gute Resultate liefern. Ich meine die Methode von Berzeurus ${ }^{2}$, die auf der Reduktion des Molybdänsäureanhydrids durch Wasserstoff beruht, und die Methode von DER Prordten, welche sich auf die Reduktion des Polysulfids gründet. Das kritische Studium dieser beiden Methoden bildet den Gegenstand vorliegender Arbeit.

\section{Methode von Berzeluus (genannt von Debray).}

Diese Methode ist modifiziert und verbessert worden nacheinander von Wöhler, Rammelsberg, Débray und Lothar Meyer und HaAs.

Sie geht allgemein unter dem Namen der Methode von Debray. In ihrer Anwendung reinigt man das Molybdäntrioxyd durch Sublimation in einem Platinapparat und reduziert dann das reine Sublimationsprodult im Wasserstoffstrom. Aber hier zeigt sich die erste Schwierigkeit. In welchem Apparat soll man arbeiten! Nimmt man Platinschiffchen, so erbält man ein platinhaltiges Molybdän und molybdänhaltige Schiffchen. Entgegen der Bemerkung von Hrn. von DER PFondten, der behauptet, dals man die Schiffchen

1 Ins Deutsche übertragen von $O$. Unger.

${ }^{2}$ Um die Litteratur über die Darstellung von Molybdän kennen za lernen, zieht man vorteilhaft zu Rat:

Dammer, Handbuch der anorg. Chem., 3, 590.

Fenuna, Handwörterbuch der Chemie, Art. Molybdän.

FrémY, Encyclopédie chimique.

Z. anorg. Chem. XI. 
durch Erhitzen bis zur Rotglut in der Oxydationsflamme und darauffolgende Behandlung mit Salpetersäure und Ammoniak leicht reinigen könne, ist es mir nicht gelungen, durch diese Behandlung das Schiffchen von Platin-Iridium, dessen ich mich bedient hatte, rein zu bekommen. Ich habe sogar gefunden, dafs nach einigen Operationen das Platin brüchig wird und bald nicht mehr zu gebrauchen ist. Ich nabm deshalb in der Folge lieber Porzellanschiffchen. Nach einer einzigen Operation ist das Schiffchen stark angegriffen und das erbaltene Produkt enthält eine beträchtliche Menge Molybdänsilicium. Das letztere kann zum grölsten Teil beseitigt werden, wenn man die am Schiffchen haftenden Teile verwirft. Das Schiffchen ist dann mit einer Metallkruste ausgekleidet und bietet für die späteren Operationen weniger Gefahr. Um die Spuren von Silicium, die das Molybdän noch enthalten kann, zu entfernen, wasche ich es mit Flulssäure, Salzsäure und destilliertem Wasser und trockne es im Vacuum.

Im folgenden gebe ich den von mir eingeschlagenen Weg zur Gewinnung von Molybdän im grofsen.

\section{Reduktion des Molybdäntrioxyds.}

Den Wasserstoff gewinnt man durch Behandlung von arsenfreiem Zink mit verdünnter Schwefelsäure. Der Entwickelungsapparat besteht aus einer grofsen, dreifach tubulierten WoulfF'schen Flasche, welche die Säure enthält. Diese Flasche trägt einen Behälter, dessen Ansatzrohr bis auf ihren Boden hinabreicht. Am oberen Ende dieses Rohres befindet sich eine Lage kleiner Porzellanscherben, die das Hinabgleiten von Zink verhüten sollen. Der Tubus $t$ gestattet die Einführung von Zink. Der Tubus $t^{1}$ ist mit dem Tubus $t^{\text {प }}$ durch ein T-Rohrsystem verbunden, welches andererseits mit einer Flasche verdünnter Schwefelsäure in Verbindung steht; diese kann behufs Regulierung des Gasdrucks höher und tiefer gestellt werden. Der Hahn, den das T-Rohr trägt, hat den Zweck, das Rohr $t^{\mathrm{HI}}$ anzusaugen.

Der so erzeugte Wasserstoff wird durch drei Thörner'sche Apparate geschickt, die nacheinander Kaliumpermanganatlösung, Natronlauge und konz. Schwefelsäure enthalten. Er passiert dann ein Rohr von böhmischem Glas, das, zwei Meter lang, nacheinander durch Kohlenoxyd reduziertes Kupfer, Platinschwamm, Phosphorsäureanhydrid und im Uförmigen Teil Glasperlen enthält, die die Verfüchtigung des Phosphorpentoxyds verhindern sollen. Das 
Kupfer und der Platinschwamm werden auf Rotglut exhitzt, und bezwecken die Zurückhaltung der letzten Spuren Sauerstoff. Das Rohr von böhmischem Glas steckt in einem Rohr von Berliner Porzellan, das $1.2 \mathrm{~m}$ lang ist und $25 \mathrm{~cm}$ inneren Durchmesser besitzt, und dessen beide Enden je einen kleinen eisernen Kühler tragen, um eine Erwärmung der Stopfen zu umgehen.

Ein absoluter Schlufs des ersten der beiden Stopfen wird dadurch gesichert, dafs man eine Hülse, die sowohl Glasrohr wie Porzellanrohr umgiebt, mit Paraffin ausgiefst. Das Porzellanrohr liegt in einem Mermar'schen Gasofen, der die Temperatur des schmelzenden Kupfers zu erreichen erlaubt. Es ist nötig, dem Ofen eine kleine Neigung za geben, um den Ablauf des bei der Reduktion entstehenden Wassers zu erleichtern. Aus demselben Grunde ist der letzte Stopfen mit einem relativ weiten $(8 \mathrm{~mm}) \mathrm{Ab}$ leitungsrohr versehen, das exzentrisch in den unteren Teil des Korkes steckt. Ein Chlorcalciumturm bildet das Ende des Apparates. In dem Porzellanrohr stehen drei Porzellanschiffchen von $9 \mathrm{~cm}$ Länge und $2 \mathrm{~cm}$ Breite, welche das ganze reine Molybdäntrioxyd enthalten. Dieses Molybdäntrioxyd entbält nur mehr Spuren von Silicium. Ich fand es unnötig, die langwierige Arbeit der Sublimation des Anhydrids zur Entfernung des Siliciums in einem Platinapparat vorzunehmen, da es wälrend der Reduktion ja wieder Silicium aufnimmt.

Bevor ich zur Reduktion schreite, leite ich durch den Apparat zwei Stunden lang einen Wasserstoffstrom, um die Luft thunlichst zu verdrängen. Das Porzellanrohr wird dann stufenweise erhitzt, bis Wasserdampf im Ableitungsrohr auftritt, und die Temperatur wird dann während einer gewissen Zeit konstant gehalten. Es vollzieht sich so eine teilweise Reduktion schon bei niedriger Temperatur, und man verhütet dadurch Verluste an Molybdänsäureanhydrid durch Sublimation. Zeigen sich Wassertröpfchen in reichlicher Menge im Ableitungsrohr, dann steigere ich die Hitze allmählich bis zum Maximum und treibe einen starken Wasserstoffstrom hindurch. Es bilden sich dann grofse Mengen Wasser, die rasch aus dem heifsen Teil des Rohres entfernt werden. Der Wasserstoffstrom wird erst verlangsamt, wenn das Ableitungsrohr trocken wird, und dann fahre ich mit dem Erhitzen noch eine Stunde fort. Hierauf lärst man das Porzellanrohr im Wasserstoffstrom abkühlen.

Jede Darstellung von Molybdän wurde morgens begonnen, während des Nachmittags führte ich die Reduktion aus, der Apparat 
kühlte sich während der Nachtstunden $a b$ und wurde erst am nächsten Morgen geöffnet.

\section{Reinigung des erhaltenen Molybdäns.}

Das so erhaltene Molybdän war von schön blaisgrauer Farbe. Es enthielt notwendigerweise Spuren von Molybdänsilicium, die von der Kieselsäure, die in dem angewandten Molybdäntrioxyd enthalten war, und zugleich von der Einwirkung des Molybdäns auf die Schiffchen herrührte. Ich habe oben angedeutet, wie ich dieses Silicium entfernen konnte.

Aber eine andere wichtige Frage erhebt sich hier. Enthält das bei so hoher Temperatur reduzierte Metall nicht noch von unvollständiger Reduktion herrührende niedrigere Oxyde? Diese Frage mufs Lothar Meyer ${ }^{2}$ vorgeschwebt baben, als er empfahl, die letzten Spuren von niedrigen Oxyden durch Exhitzen des Molybdäns im Chlorwasserstoffstrom zu entfernen. So schrieb er in einem Bereicht über die Arbeit von LiEchmr und REMPE ${ }^{2}$, welche das Studium der Chloride des Molybdäns durchgeführt hatten:

„Die Hauptschwierigkeit, der Blomstraud nicht ganz zu begegnen wuiste, bestand in der Beschaffung oxydfreien Molybdäns. das durch Reduktion nicht leicht erhalten wird. Man gewinnt dasselbe sehr leicht, wie ich schon vor mehreren Jahren in Gemeinschaft mit Herrn RoBERT HaAs von hier, jetzt in Irun, beobachtete, wenn man das unvollständig reduzierte Metall in trockner Chlorwasserstoffsäure erhitzt, wobei sich alles Oxyd in die von Débray entdeckte leicht flüchtige weilse Verbindung $\mathrm{MoO}_{3}$. $2 \mathrm{HCl}$ verwandelt, die nach der Analogie der aus $\mathrm{SO}_{3}$ und $\mathrm{HCl}$ entstehenden Verbindung $\mathrm{SO}_{2} \mathrm{OHCl}$ wahrscheinlich als ein Hydroxylchlorid des Molybdäns $\mathrm{Mo}(\mathrm{OH})_{2} \mathrm{Cl}_{2}$ anzusehen ist."

Nach diesen Autoren genügt es also, das oxydhaltige Molybdän in einem Salzsäurestrom zu erhitzen, um allen Sauerstoff in der Form von $\mathrm{MoO}_{3} .2 \mathrm{HCl}$ zu entfernen.

Um zu prüfen, inwieweit diese Behauptung von MEYER und HaAs richtig ist, habe ich ungefähr $20 \mathrm{~g}$ Molybdän bei $500^{\circ} \mathrm{C}$. in eine trockene Salzsäureatmosphäre gebracht. Ich erhitzte das mit dem Molybdän beschickte Rohr von böhmischem Glas erst, als das Salzsäuregas, das den Apparat passiert hatte, von Wasser voll-

' Lothar Mever, Ber. deutsch. chem. Ges. 6, 991.

${ }^{2}$ Licnix und Rempe, Ann. Chem. Pharm. 169, 344. 
ständig absorbiert wurde. Indem ich, wie angegeben, verfubr, was ich sehr überrascht, wie jch sah, dafs aus dem zur Absorption des Salzsäuregases bestimmten Wasser Gasblasen entwichen. Das Gas hätte Luft oder besser Stickstoff sein können, wenn man annahm, dais das Molybdän Sauerstoff gebunden enthielt. Wie grofs war mein Erstaunen, als ich fand, dafs das aufgefangene Gas Wasserstoff ${ }^{1}$ war!

Ich glaubte einen Augenblick, dals das Molybdän diesen Wasserstoff durch Adsorption bei seiner Bereitung festgehalten hatte. Folgendes Experiment hat mich eines Besseren belehrt. Ich verbrannte ca. $1 \mathrm{~g}$ Molybdän in einem Rohr, das mit einem Apparat von VOLHARD verbunden war. Letzterer änderte sein Gewicht nicht. Ich werde später im einzelnen auf die Versuche eingehen, die beweisen, dafs Wasserstoff auf Molybdän ohne Einwirkung ist.

Die Entbindung von Wasserstoff, von der weiter oben die Rede war, könnte durch die Gegenwart von Spuren Molybdänsilicium erklärt werden. Indessen ist zu bemerken, dafs diese Gasentwickelung bei $200^{\circ}$ nicht auftrat. Es läfst sich nun aber vermuten, dals das Molybdänsilicium durch die Salzsäure nach Mafsgabe der Wärme zersetzt würde. Es wäre auch möglich, dals bei der von mir eingehaltenen Temperatur das Molybdän oder seine niederen Oxyde unter Wasserstoffentbindung chloriert werden. Schlofs endlich das Salzsäuregas nicht Spuren von Feuchtigkeit in sich, die entweder von der unvollständigen Trocknung durch Schwefelsäure ${ }^{2}$ oder von der Einwirkung des Gases auf das Glas herrührte?

Diese Frage kann man nur dann aufklären, wemn die Abwesenheit von Oxyden im Molybdän absolut sicher ist.

Es ist noch zu bemerken, dafs nach der Behandlung des Molybdäns mit gasförmiger Salzsäure und Abkühlung des Apparates das Rohr durch trockene Luft sorgfältig von Salzsäuregas befreit worden war, bis die Luft keine saure Reaktion mehr gab. Trotz

' Ich hatte diese Erscheinung schon beobachtet, als ich feinverteiltes Molybdän dieser Behandlung unterwerfen wollte. Letzteres war aus Ammoniummolybdat, mit dem ein gereinigter Bimsstein getränkt worden war, durch Glühen und Reduktion erhalten worden. Hier konnte die Wasserstoffentwickelung von Molybdänsilicium herrühren, das bei der Einwirkung der Salzsäure auf den Bimsstein entstand.

${ }^{2} \mathrm{P}_{2} \mathrm{O}_{5}$ konnte zum Trocknen des Gases keine Verwendung finden, da sich flüchtiges Phosphoroxychlorid bildete. 
dieser Vorsichtsmafsregeln gab das Molybdän unzweideutig die Reaktion auf Chlor!

Um die experimentelien Bedingungen einhalten zu können, unter denen L. MeYer und HaAs gearbeitet hatten, wandte ich mich an ersteren. Hr. Lothar MEYER war so gütig, in einem rom 23. Nov. 1893 datierten Brief folgendermalsen zu antworten:

„Die von Thnen gewünschte Auskunft gebe ich Thnen sehr gerne. Wie Sie schon richtig vermuten, ist die Dammer'sche Angabe, ${ }^{1}$ dafs man glühen solle, ganz irrtümlich, man braucht nur gelinde zu zu erhitzen, ganz wie Drkray angiebt, ob wir aber die Temperatur gemessen haben, erinnere ich mich nicht mehr. Herr $\mathrm{H}_{\text {AaS }}$ hat seine Arbeit nicht weiter geführt, und ich habe über dieselbe nichts weiter veröffentlicht, als die Ihnen bekannte Notiz"."

Ich nahm damn die Versuche nach den Angaben von Lothar MEYER wieder auf, indem ich in einer Salzsäure-Atmosphäre auf $200^{\circ}$ erhitzte. Das Molybdän war vorläufig gereinigt worden durch successives Waschen mit Fluissäure, Salzsäure und destilliertem Wasser in einer Platinschale. Als nach längerem Waschen mit destilliertem Wasser das Molybdän keine Chlorreaktion mehr gab, trocknete ich es im luftleeren Raum.

Ungefähr drei Gramm dieses Molybdäns sind in den Apparat in gasförmige Salzsäure eingeführt worden. Die Temperatur wurde bei $200^{\circ}$ gehalten. Es bildete sich im Anfang ein leichter weilser Beschlag. Nach dem Verlauf einer Stunde hatte jede Sublimation aufgehört, aber die Operation wurde noch eine Stunde fortgesetzt. Nach dem vollständigen Erkalten des Apparates liefs ich zwei Stunden lang einen Strom trockene Luft hindurch passieren. Dann wurde die das Schiffchen enthaltende Röhre luftleer gemacht und abermals während einer halber Stunde ein Luftstrom hindurch geschickt. Ein halbes Gramm dieses Molybdäns wurde nun mit $10 \mathrm{ccm}$ destilliertem Wasser angerührt. Nach Verlauf einer Minute fiel das Metall auf den Boden der Reagensröhre; die überstehende Flüssigkeit wurde abdekantiert, mit einigen Tropfen Salpetersäure angesäuert und gab eine deutliche Chlorreaktion!

Diese Versuche zeigen klar, dafs die Behandlung des Molybdäns mit gasförmiger Salzsäure, weit entfernt, es zu reinigen, dasseibe

\footnotetext{
1 DAMMEk, a. a. O.
}

2 L. Mever, a. a. $O$. 
mit Chlor verunreinigt, also nicht als Reinigungsmethode angesehen werden kann.

Diese Behandlung ist auch ganz unzureichend zur Entfernung allen Sauerstoffes, den das Molybdän in Form von Oxyden enthalten könnte - trotz der Angaben von L. MeYeR und HAAS, wie ich sogleich zeigen werde.

\section{Einwirkang der Salzsäure auf teilweise oxydiertes Molybdän.}

Molybdän, das mit Flufssäure, Salzsäure und destilliertem Wasser gewaschen und dann im Vacuum getrocknet worden war, wurde durch Erhitzen an der Luft teilweise oxydiert. Die Gewichtszunahme gab die Menge des aufgenommenen Sauerstoffes an. Die freie Oberfläche zeigte einen braunen Fleck, der von der Bildung eines höheren Oxydes herrührte. Dieses partiell oxydierte Molybdän wurde in einem Salzsäurestrom auf $200^{\circ}$ erhitzt. Gleich am Anfang zeigte sich ein schwaches weifses Sublimat. Die Operation wurde unterbrochen, als die Sublimation ganz aufgehört hatte.

Nachstehend die Resultate dieser Untersuchung.

$$
\begin{array}{ll}
\text { Angewandtes Molybdän } & 0.7775 \mathrm{~g} . \\
\text { Gefundener Sauerstoff } & 0.042 \mathrm{~g} .
\end{array}
$$
$0.063 \mathrm{~g}$.

Gefundener Gewichtsverlust nach der Behandlung mit HCI-Gas bei 200"

Berechneter Gewichtsverlust, unter der Annahme, dafs der gebundene Sauerstoff das Molybdän in Form der Verbindung $\mathrm{MoO}_{3} .2 \mathrm{HCl}$ verläfst $0.126 \mathrm{~g}$.

Hierauf oxydierte ich die nämliche Probe Molybdän wieder, indem ich die Oxydation weiter trieb, dann nahm ich die Behandlung mit Salzsäuregas bei $200^{\circ}$ wieder auf.

$\begin{array}{ll}\text { Molybdän + Spuren von Molybdänoxyds } & 0.7565 . \\ \text { Während der zweiten Oxydation gebundener Sauerstoff } & 0.1085 . \\ \text { Beobachteter Gewichtsverlust nach der Behandlung mit HCl-Gas } & 0.091 . \\ \text { Berechneter Gewichtsverlust aus dem neugebundenen Sauerstoff } & 0.3255 .\end{array}$

Ich wiederholte die Oxydation ein drittes Mal, indem ich den Rückstand der zweiten Behandlung mit Salzsäuregas auf $500-600^{\circ} \mathrm{C}$. erhitzte. Es bildete sich wiederum ein weilser Bescblag. Als die Sublimation ganz aufgehört hatte, machte ich eine dritte Wägung.

Beobachteter Gewichtsverlust beim Erhitzen auf $500-600^{\circ}$ C. 0.2365 .

Das Molybdän hatte die braunen Flecken noch. Dies zeigt schon allein, dafs die Salzsäure aufser stande ist, dem Molybdän die ihm anbängenden Spuren Sauerstoff zu entziehen. 
Kurz zusammengefaist, habe ich festgestellt, dals das durch Reduktion des Molybdänsäureanhydrids mit Wasserstoff dargestellte und mit Flufssäure und destilliertem Wasser gewaschene Molybdän nach der Behandlung mit Salzsäuregas bei $200^{\circ}$ die Reaktion der Chloride giebt. Bei höherer Temperatur bildet sich unabhängig von dem weifsen Sublimat Débray's eine rote Substanz, die ich als Oxychlorid anspreche, und Wasserstoff wird entwickelt.

Aufserdem habe ich gefunden, dafs nach obigen Angaben gereinigtes und dann einer partiellen Oxydation unterworfenes Molybdän durch Einwirkung von trockener Salzsäure nicht von allen Oxyden, die es enthält, befreit werden kann.

Es ist möglich, dafs gewisse Oxyde des Molybdäns das Sublimat von Drbray's $\mathrm{MoO}_{3} .2 \mathrm{HCl}$ liefern können.

Ein zusammengesetztes Oxyd, z. B. von der Formel

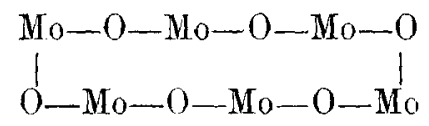

könnte sich leicht in $4 \mathrm{Mo}+2 \mathrm{MoO}_{3}$ umlagern, welch letzteres sich dann mit $4 \mathrm{HCl}$ verbinden würde. Aber meine Versuche beweisen, dals nicht alle Oxyde sich so verhalten und fähig sind, sich in einer Salzsäureatmosphäre in $\mathrm{nMo}+\mathrm{m} \mathrm{MoO}_{3} \cdot 2 \mathrm{HCl}$ zu spalten.

Es wäre interessant, die Einwirkung der gasförmigen Salzsäure auf alle wohldefinierten Oxyde des Molybdäns zu studieren. Ich habe diese Aufgabe für das krystallisierte Molybdändioxyd unternommen, das man durch Schmelzen eines Gemenges von Ammoniummolybdat und Molybdäntrioxyd mit Borax und Kaliumcarbonat erhält. ${ }^{1}$ Ich fand, dals bei diesem Oxyd gegen $200^{\circ}$ ein leichter weifser Beschlag auftritt, der wahrscheinlich von einer Spur Molybdänsäureanhydrid herrührt; aber das Aussehen des Dioxyds ändert sich durchaus nicht, selbst wenn man es sehr stark erhitzt. Diese Arbeit wird den Gegenstand einer späteren Veröffentlichung bilden.

Die zahlreichen Versuche, die ich angestellt habe, haben mich überzeugt, dafs die Reinigung des Molybdäns durch trockene Salzsäure nur das Molybdänsäureanhydrid entfernen oder die Oxyde zerlegen kann, welche als Molybdänmolybdate angesehen werden können. Die Methode von L. Mexer und HaAs beseitigt die niedrigen Oxyde nicht. Für alle Anwendungen, wo die Gegenwart der:

${ }^{1}$ Muthmaxy, Ann. Chim. Pharn. 238, 116. 
letzteren keine Unzuträglichkeiten bereitet, giebt die DrBRAY'sche Methode ein genügend reines Produkt.

\section{Methode von von der Pfordten.}

VON DER PFondTEN ${ }^{1}$ entdeckte eine neue Methode für die Bereitung von Molybdän, die sich auf die Reduktion des Polysulfids durch Wasserstoff gründet. Indem er die Arbeiten von L. MeYer und HaAs, Liechit und Rempe ins Gedächtnis zurückruft, sagt er: „Eine ähnliche Malsregel ist bei der Reduktion der Sulfide nicht nötig, auch kann man sich leicht davon überzeugen, ob das erhaltene Produkt gänzlich schwefelfrei ist." Der Verfasser behauptet, dafs die Reduktion vollständig ist und dafs seine Methode sogar zur quantitativen Bestimmnng der Molybdänsäure dienen kann. Von dem Wunsche getrieben, ein ganz sauerstofffreies Molybdän zu erhalten, benutzte ich seine Methode. $\mathrm{Zu}$ diesem $\mathrm{Zwecke}$ wurde eine durch Zusatz eines grolsen Überschusses von Schwefelammonium. zu Ammoniummolybdat erhaltene Lösung von Sulfomolybdat durch Salzsäure zersetzt. Der so gewonnene Niederschlag von Molybdänpolysulfid und Schwefel wurde mit gesättigtem Schwefelwasserstoffwasser gewaschen und in einem Schwefelwasserstoffstrom, der durch eine gesättigte Natriumsulfidlösung gegangen und so gereinigt ${ }^{2}$ worden war, bei $100^{\circ}$ getrocknet.

Ich arbeitete mit ungefähr $15 \mathrm{~g}$ trockenem Polysulfid. Diese führte ich in das Porzellanrohr des Wasserstoffapparates (Fig. 1) ein, indem ich mit allen Vorsichtsmalsregeln arbeitete, die ich bei der Reduktion des Molybdäntrioxyds angewandt hatte. Ich unterbielt den Wasserstoffstrom durch 24 Stunden, bei der höchsten Temperatur, die der Ofen leistete. Es entwickelt sich stetig Schwefelwasserstoff, selbst wenn man glauben sollte, dals die Reduktion vollendet ist. Es ist nicht anders zu erwarten, denn an gewissen Stellen des Rohres muls sich ein Zustand bilden, der durch das Schema: $\mathrm{H}_{2} \mathrm{~S} \longleftrightarrow \mathrm{S}+\mathrm{H}_{2}$ ausgedrückt wird.

Da ich, nach 24 Stunden, erwarten konnte, dafs die Reduktion vollständig war, liefs ich den Apparat erkalten. Aber ich fand, dafs das Molybdän noch Schwefel enthielt. Ich fing deshalb in einem Wasserstoffstrom wiederum 24 Stunden zu erhitzen an und fand nach dieser Operation noch Spuren von Schwefel in dem Produkt.

\footnotetext{
1 Von der Pfordten, Ber. deutsch. chem. Ges. 17, 732.
}

2 Stas, Ges. Werke. 1, 849. 


\section{4}
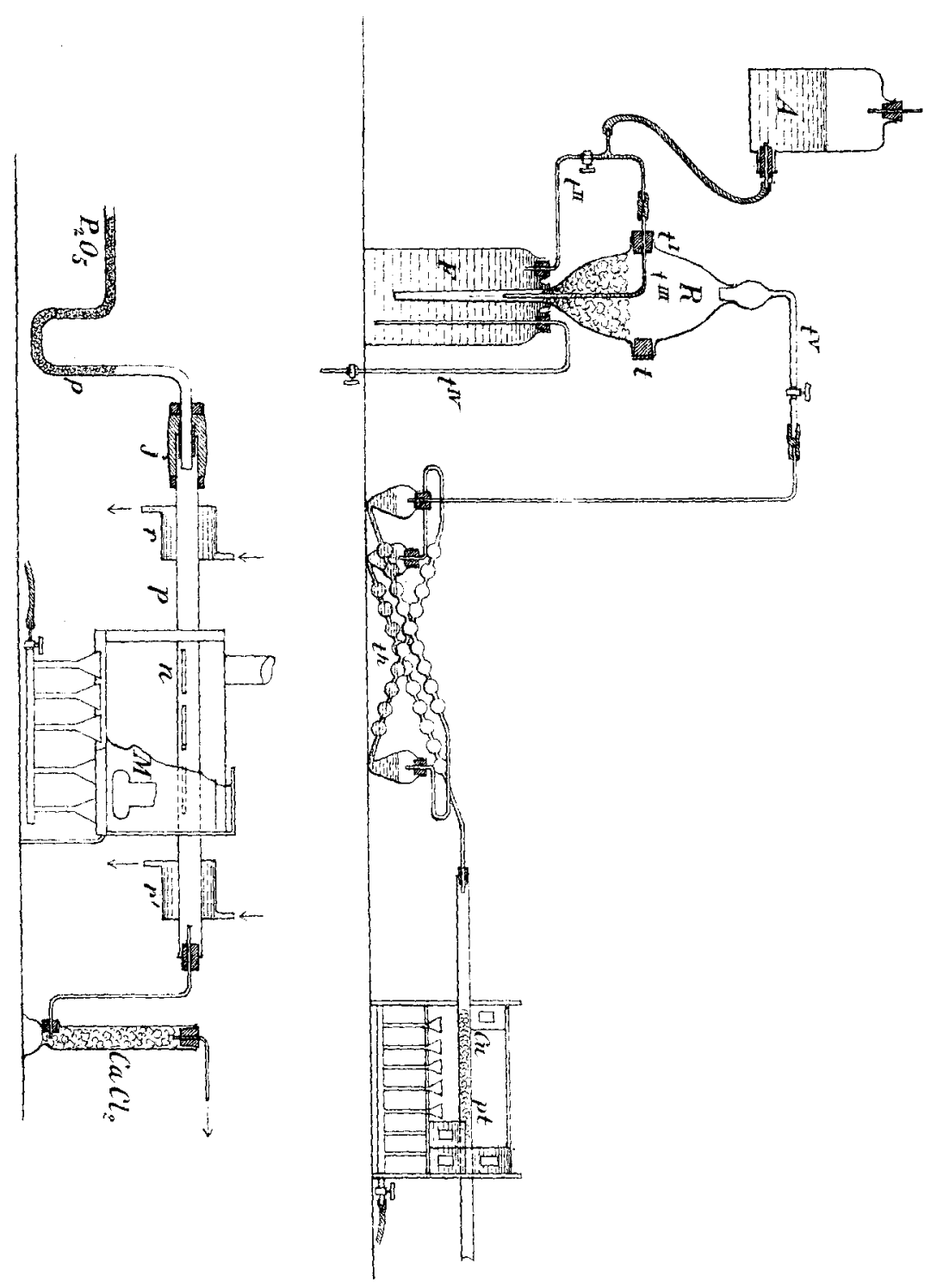

Fig. 1 (Wasserstoffapparat).

$A R F, T-T v$ Entwickelungsapparat. th 3 'ThönNen'sche Apparate für die Lösungen von Kaliumpermanganat, Âtzkali und konz. Schwefelsäure. Cu Kupfer, durch Kohlenoxyd reduz. $P t$ Platinschwanne.
$P_{2} O_{5}$ Phosphorpentoxyd. $p$ Glasperlen.

j Paraffinverschlufs.

$r r^{\prime}$ Kühler, $\quad P$ Porzellanrohr.

$M$ Mermetscher Ofen.

$\mathrm{CaCl}_{2}$ Chlorcalciumturm. 
Ich muls glauben, dafs die Methode von von DER Pfordoten nur genügend ist, wenn es sich darum handelt, im Gang einer Analyse einige Centigramme Molybdänsulfid zu zersetzen. ${ }^{1}$ Sie scheint mir nicht mehr die Eigenschaften einer Darstellungsmethode zu besitzen.

\section{Das Molybdän von STERnBerg und DeUTSCH.}

Man findet im Handel ein Metall, das unter dem Namen: "Molybdän von Stmenberg und DevTscr" käuflich ist. Dieses Molybdän wird durch Reduktion von Calciummolybdat durch Kohle erhalten. Es bildet ein sehr feines, tiefgraues Pulver.

Ich habe ron diesem Produkt eine Bauschanalyse gemacht.

Ich nahm $10.166 \mathrm{~g}$ Substanz. Nach mehrstündigem Verweilen im Trockenkasten bei $105^{\circ}$ ergab sich ein Gewichtsverlust von $0.063 \mathrm{~g}$, der wahrscheinlich einem Weggang von Feuchtigkeit zuzuschreiben ist. Aber nach 24 stündigem Erhitzen fand ich eine Gewichtszunahme von $0.02 \mathrm{~g}$ und nach 28 Stunden betrug sie 0.105 g. Diese Gewichtszunahme rührt sicherlich von der Oxydation des Molybdäns oder niedriger Oxyde her, die dieses Produkt enthalten kann. Es war von vornherein anzunehmen, dafs dieses Molybdän Kohle enthielt. Zur Bestimmung ihrer Menge verfuhr ich wie bei einer organischen Analyse. Ich fand $8 \%$ Kohlenstoff in einer Portion, die bei $100_{0}$ getrocknet war.

\section{Das Molybdän von MoIssan.}

MoIssan hat reines geschmolzenes Molybdän erhalten, ${ }^{2}$ indem er das Gemenge von Molybdänoxyd und Kohle der Hitze eines

${ }^{1}$ Es folgt übrigens aus den vom Autor selbst gegebenen Daten, dals die Reduktion auch bei Anwendung kleiner Quantitäten Sulfid nicht vollständig ist.

Angewandte Substanz
(natürliches Bisulfid):
I. $0.1488 \mathrm{~g}$
II. $0.2932 \mathrm{~g}$
III. $0.3973 \mathrm{~g}$

Gefundene Menge Schwefel:
I. $32.3 \%$
II. $36.1 \%$
III. $39.5 \%$

${ }^{2}$ H. Moissan, Compt. rend. 120, 1320.

$$
\begin{aligned}
& \text { Dauer } \\
& \text { der Reduktion: } \\
& 40 \text { Min. } \\
& 11 / 2 \text { Stdn. } \\
& 3 \text { Stdn. }
\end{aligned}
$$

Berechnete Menge:

$$
\begin{aligned}
& 40 \% \\
& 40 \% \\
& 40 \%
\end{aligned}
$$


durch einen Strom von 800 Amp. und 60 Volt erzeugten Flammenbogens 6 Minuten lang aussetzte.

Wenn man annimmt, dals das Molybdän nicht noch Spuren niedrigerer Oxyde einschliefst - was noch $\mathrm{zu}$ beweisen ist -, folgt aus vier von Modssan ausgeführten Analysen, dafs sein reines geschmolzenes Molybdän 0.08-0.28\% Verunreinigungen enthält.

Es existiert also bis jetzt keine Darstellungsmethode für chemisch reines Molybdän. Es ist mir gelungen, diese Lücke zu füllen, durch eine bis jetzt nicht beschriebene Methode, die den Gegenstand einer besonderen Untersuchung bilden wird.

Das Prinzip dieser Methode ist niedergelegt in einem verschlossenen Kouvert, das ich die Ehre hatte der Kgl. Belgischen Akademie der Wissenschaften anzuvertrauen.

Im Laufe dieser Untersuchungen bin ich dazu geführt worden, die Einwirkung verschiedener Gase, wie Wasserstoff, Stickstoff und Kohlendioxyd auf erhitztes Molybdän zu studieren. Die Resultate dieser Untersuchungen bilden den Gegenstand der folgenden $\mathrm{Ab}$ handlung. 